\title{
RECENT ADVANCES IN BACTERIOLOGY
}

\author{
N. PAUL HUDSON, \\ Department of Bacteriology, The Ohio State University
}

As is true of many sciences in this period of scientific advancement, Bacteriology has extended its frontiers in many directions. In so doing Bacteriology has joined hands with numerous scientific neighbors with the result that subject boundaries have become indistinct, and many areas are held jointly by two or more sciences. Furthermore, the advanced nature of modern research requires a mutual exchange of techniques among sciences and demands their close association in research for the solution of the problems that now present themselves for study. The circumstances of the highly technical war that we have just waged have shown us how necessary and effective is this teamwork among sciences.

Things being as they are, then, advances in Bacteriology are not circumscribed by limitations implied by the term "Bacteriology," but are associated with progress in allied fields. During the war, many advances were particularly concerned with the applications of scientific knowledge on a large scale.

With this understanding, let us briefly consider the advances recently made in four fields arbitrarily designated as (1) General Bacteriology, (2) Medical Bacteriology and its Applications, (3) Viruses, and (4) Medical Mycology.

(1) General Bacteriology. - For some years the sulfa drugs have been studied and employed as chemotherapeutic agents, with results known to all. Bacteriology has developed the techniques of experimental evaluation and of the control and interpretation of clinical trial. The mode of action of sulfa drugs was early recognized as a problem of bacterial metabolism, and while the explanation is still theoretical and not always applicable, it is now most generally thought to be of this sort: bacteria that are sensitive to sulfa drugs owe this sensitivity to the facts, first, that they, like most other living things, require vitamines or vitamine-like substances for their growth and development; second, that they are unable to distinguish between these essential substances and structurally similar compounds such as the sulfa drugs; and third, once having taken up the metabolically inert sulfa molecule the bacterial cell is incapable of utilizing the growth factors. Bacteria thus held in a condition of stasis readily succumb to the defense mechanisms of the host.

Antibiotics, those substances produced by molds and bacteria and antagonistic to other microorganisms, are likewise well known as therapeutic agents. Bacteriology has established methods of laboratory assay and by such means can tell the concentration of an antibiotic in the body fluids of patients. The mode of 
action of this biological product is as yet undefined, but it does appear that the antibiotics now in use exert their effects by bacteriostasis when the concentration of the drug is low and by bacteriocidal and bacteriolytic actions when the drug is is high concentration.

For some time it has been known that the types of pneumococcus are differentiated according to the specific carbohydrate composition of the capsule, and that a pneumococcus could be changed from one type to another by certain laboratory procedures. The interesting observation has recently been made that exposure to desoxyribonucleic acid from an encapsulated Type III transforms an avirulent non-encapsulated Type II into a fully virulent Type III pneumococcus. In this way, one variety of bacterium is converted to another by the presence of a substance of known chemical composition, not unlike the determining action of a gene in higher forms.

These instances of alteration of bacterial metabolism by the presence of certain substances point to the so-called "phenomenon of interference" in bacterial physiology. With this phenomenon brought to the attention of investigators, other examples are constantly being described having to do with the competition of bacteria and viruses for food elements, some of which are chemically defined. This type of work involves a detailed chemical and biological approach to the study of enzymes, bacterial metabolism and parasitism and will yield fruitful results with further exploration and analysis.

Advances in biophysics, as applied to Bacteriology, are only begun, but already there is much promise in the use of tagged and radioactive elements in the study of bacterial metabolism. It is expected that the utilization of tagged elements will disclose how molecular chemical reactions are brought about by bacteria and how the chemical structure of bacteria is built.

The electron microscope has been used to explore, by its high magnification, the finer details of bacterial composition and the shape and size of viruses. Attention has been concentrated on the finding of minute structures in bacteria and the possibility of their representing nuclear elements. Thus far, no definite nucleus or chromosomal mechanism has been established, but the interpretation of the physical findings combined with histo-chemical studies may shed light on this basic and baffing question in bacterial morphology and genetics.

(2) Medical Bacteriology and Applications.- It is unnecessary to dwell on the valuable improvement of diagnostic methods by the laboratory workers of the Armed Forces. Not only has the best effort of highly qualified experts been spent on this practical question, but also the circumstances of military activities in so widely scattered parts of the world have necessitated a development of techniques in microbiological fields hitherto not possible.

The control of air-borne infections has been studied intensively by military and civilian groups. The scientific approach has consisted of the development and application of methods for the determination and control of the factors involved in transmission of respiratory bacteria, such as particle size, conditions favoring suspension and transmission of dust and respiratory droplets laden with bacteria, devices to sample air and to count its contained bacteria, methods of dispensing disinfecting aerosols in barracks, schools and other common rooms, the efficiency of germicidal light, and the oiling of floors and bedding to keep down contaminated dust. Above all, much effort has been spent on the interpretation of the results of using one or another of the many devices devised. This whole program has had and will continue to have far reaching results in the difficult field of respiratory infections.

Medical Bacteriology has been much concerned with drug fastness to sulfa drugs and antibiotics. Too often for completely satisfactory results, bacteria either in a single patient or passed from person to person have proved by bacteriological methods to be resistant to the drugs in use. This seems to be more a 
matter of selection and survival of drug-fast bacteria in a bacterial population than the sudden conversion of a whole population to the state of resistance. Once the fastness fortuitously occurs, it persists in the strain transmitted to subsequent hosts. Medical bacteriological laboratories now use techniques for the determination of such fastness and report the findings to the clinician or public health official who takes the observation into account in further management of the situation.

The applications of Medical Bacteriology would not be completely recounted without a reference to the improvement and probably increased effectiveness of certain bacterial vaccines, such as those against typhoid and paratyphoid fevers, cholera, plague and tetanus. Also, reference should be made to the measures taken to combat hazards of water-borne infections and bacterial food poisoning experienced by our men under conditions of combat and service in the near and remote parts of the world from the Equator to the Arctic Circle. Here, methods of sanitary control, preparation of safe food, vaccines and education reduced greatly the likelihood of food and water-borne disease. Similarly, the development and application of insecticides were instrumental in reducing the incidence of insect-borne disease.

(3) Viruses.-The study of viruses and virus diseases, well advanced before the war, was given a marked impetus by the necessities of military activity. The solution of earlier problems was advanced, such as in influenza in which laboratory diagnostic methods were improved, epidemiology studied, and vaccines developed and applied. Similarly, typhus received much attention with the result that serological tests were devised, the relation of epidemic typhus of man and endemic typhus of rats was more clearly defined, and the typhus vaccine was improved. Attention to scrub typhus, met by our men in the Far East and Western Pacific, necessitated intensive studies that disclosed the native rodents as sources of infection, insects as vectors, and the elimination of these factors in camp sites as measures of control. Progress has also been made in the development of vaccine.

Atypical pneumonia and infectious hepatitis occurred seriously during the war, the former in increased incidence and the latter in epidemic form. The specific cause of atypical pneumonia is as yet undetermined, although bacteria are thus far excluded. It is interesting that so many cases should be recognized clinically in recent years, as if it were a new disease, and yet no one virus of several proposed has convincingly been shown to be the sole specific cause. Infectious hepatitis, first seen arising from a contamination in the human serum component of yellow fever vaccine, proved serious also in large spontaneous epidemics. Lacking a susceptible experimental animal, human volunteers have been used in studies on etiology, and it now looks as though the disease is due to a virus present in patients' feces and entering by the mouth. The etiologic agent may be related to those of catarrhal jaundice and of hepatitis occasionally following injections of human serum.

Recent advances in the study of poliomyelitis have not settled the question of the modes of transmission and entry of virus into the human body. Detailed epidemiological studies, histological examinations of nervous tissues, and the finding of virus in the upper respiratory tract coincident with the infectious period of persons for others point to a substantiation of the theory of contact and droplet methods of transmission, as opposed to the theory of intestinal source and entry of virus.

The classification of viruses has been a troublesome question for those who desire to arrange their subject matter in orderly fashion. It seems now as though, by our continued detailed study of the various features of viruses and virus diseases, that at least four groups can be identified: viruses of some forms of encephalitis (St. Louis, Japanese B, Russian Spring-Summer, and louping ill); viruses of relatively large size and of similar antigenicity (psittacosis, lymphogranuloma venereum, mouse pneumonitis, and meningo-pneumonitis); viruses of pox diseases (smallpox, 
vaccinia, swine pox, etc.); the bacteriophage group; and viruses parasitizing plants. Each group is defined by reference to various criteria, such as host, antigenicity, modes of transmission, pathology, and susceptibility to sulfa drugs.

Although some viruses may be grouped according to similar characters, virologists are still more impressed by the constantly increasing heterogeniety observed in the properties of viruses, so that they may be thought of as being as varied and dissimilar as the wide range of bacteria.

(4) Medical Mycology.-Mycology has developed in relation to Bacteriology in two directions, namely, in the findings of antibiotics active against microorganisms and in the study of infections due to pathogenic fungi. Having already referred to the problems of range of bacterial antagonism, mode of action, drug fastness and biological assay, let us here mention the advances made in connection with antibiotic production itself: the discovery of new fungi elaborating the antibiotic principle, the choice of strains by careful test, the physiology of fungi, the factors affecting biological variation as exemplified by antibiotic production, purification and separation of the active principle, tests for and control of toxicity for host cells, development of media and procedures for commercial production, and the chemistry of the antibiotic preparations. Each point mentioned represents new, basic and practical advances pressed for war and scientific purposes, with results known and anticipated for the relief of suffering and the prolongation of life.

There occurs a disease in the dry regions of the Southwest due to a fungus, Coccidioides immitis. It seems to have its habitat in the soil or vegetation and when inhaled by man causes a condition which has not attracted much attention until our troops stationed in those parts for desert training became manifestly infected. This finding and the study of the fungus and disease have led to the recognition of the importance of the condition. Likewise, it has stimulated interest in other infections due to pathogenic fungi hitherto considered rare and unimportant. Such infections lead to a hypersensitivity to the proteins of the fungus and recently a large part of the young adult population in the Midwest has been found to be hypersensitive by skin test to the proteins of another pathogenic fungus, Histoplasma capsulatum. The significance of this finding is yet to be determined, but it does appear as though man even in the temperate zone possibly suffers generalized infections due to one or another fungus, in addition to the fungi that now are known to parasitize his skin, hair and nails.

These fungal infections, together with the opening field of antibiotics and the wide experience of our Armed Forces with mycotic conditions in the tropics, have served to stimulate much interest in mycology and already distinct advances have been made. Our chief lack of basic information in this field seems to be in the physiology of fungi, and we may look forward to developments in fundamental as well as in medical and industrial mycology.

To summarize, a few general remarks may be made. The basic science of Bacteriology is developing in many directions. There is no line of distinction between its various fields, nor between the diverse phases of the subject and other fields of science. Industrial and medical research in microbiology has been fundamental and abstract as well as applied, and, under whatever aegis, scientific advances have been mutually contributory. The distinction between so-called pure and applied science is fast fading if it has not indeed already disappeared.

Recent advances have contributed to another common principle, namely, the similarity of basic biological phenomena among the wide range of microscopic forms: metabolism in terms of enzymes and chemistry, cellular biology, chemical composition, parasitism, immunology, and reaction to external conditions.

If the two principal advances in Bacteriology were to be given, they might well be stated as the demonstration that scientific progress can best be made by a correlation of scientific approaches and the establishment of the common fundamental nature of the minute forms of life. 